\title{
Relationship between coronary flow reserve evaluated by phase-contrast cine cardiovascular magnetic resonance and serum eicosapentaenoic acid
}

Shingo Kato ${ }^{1 *}$, Kazuki Fukui ${ }^{1}$, Junko Kawaguchi ${ }^{2}$, Nao Ishii ${ }^{1}$, Masashi Koga', Yuka Kusakawa', Ikuyoshi Kusama', Tatsuya Nakachi ${ }^{1}$, Takeshi Nakagawa' ${ }^{1}$, Yasuo Terauchi ${ }^{2}$, Kazuaki Uchino ${ }^{3}$, Kazuo Kimura ${ }^{4}$ and Satoshi Umemura ${ }^{3}$

\begin{abstract}
Background: Long-term intake of long-chain n-3 polyunsaturated fatty acids ( $\mathrm{n}-3$ PUFAs), especially eicosapentaenoic acid (EPA) is associated with a low risk for cardiovascular disease. Phase-contrast cine cardiovascular magnetic resonance (PC cine CMR) can assess coronary flow reserve (CFR). The present study investigates the relationship between CFR evaluated by PC cine CMR and the serum EPA.

Methods: We studied 127 patients (male, 116 (91\%); mean age, $72.2 \pm 7.4$ years) with known or suspected coronary artery disease (CAD). X-ray coronary angiography revealed no significant coronary arterial stenoses (defined as luminal diameter reduction $\geq 50 \%$ on quantitative coronary angiogram (QCA) analysis) in all study participants. Breath-hold PC cine CMR images of the coronary sinus (CS) were acquired to assess blood flow of the CS both at rest and during adenosine triphosphate (ATP) infusion. We calculated CFR as CS blood flow during ATP infusion divided by that at rest. Patients were allocated to groups according to whether they had high $(n=64, E P A \geq 75.8 \mu \mathrm{g} / \mathrm{mL})$ or low $(n=63$, EPA $<75.8 \mu \mathrm{g} / \mathrm{mL}$ ) median serum EPA.
\end{abstract}

Results: CFR was significantly lower in the low, than in the high EPA group ( $2.54 \pm 1.00$ vs. $2.91 \pm 0.98, p=0.038)$. Serum EPA positively correlated with CFR $(R=0.35, p<0.001)$. We defined preserved CFR as $>2.5$, which is the previously reported lower limit of normal flow reserve without obstructive CAD. Multivariate analysis revealed that EPA is an independent predictor of CFR > 2.5 (odds ratio, 1.01; 95\% confidence interval, $1.00-1.02, p=0.008$ ).

Conclusions: The serum EPA is significantly correlated with CFR in CAD patients without significant coronary artery stenosis.

\section{Background}

Long-term intake of long-chain n-3 polyunsaturated fatty acids (n-3 PUFAs), especially eicosapentaenoic acid (EPA), is associated with a low risk for cardiovascular disease [1-7]. Several reports describe that n-3 PUFAs confer several benefits, such as antiarrhythmic effects $[8,9]$ and the ability to reduce platelet aggregation $[10,11]$ and stabilize coronary arterial plaque [12]. The GISSIPrevenzione trial [13] revealed that dietary n-3 PUFA

\footnotetext{
* Correspondence: shingo.m12226@gmail.com

'Department of Cardiology, Kanagawa Cardiovascular and Respiratory Center, 6-16-1 Tomiokahigashi, Kanazawa-ku, Yokohama, Kanagawa 236-0051, Japan
} Full list of author information is available at the end of the article intake significantly prevented cardiovascular mortality in patients with a history of myocardial infarction. The Japan EPA Lipid Intervention Study (JELIS) [14] showed that concurrent therapy with purified EPA and statins reduces the incidence of coronary events.

Phase-contrast cine cardiovascular magnetic resonance (PC cine CMR) is a promising approach to quantifying global myocardial blood flow in the left ventricular (LV) myocardium without exposure to radiation [15-19]. The accuracy of this technique has been validated in phantoms [20], in animals using flow probes [18] and in humans using positron emission tomography (PET) [16]. Coronary flow reserve (CFR) calculated from CMR flow

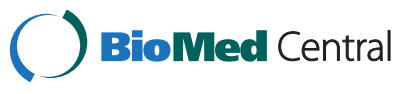


values in the coronary sinus at rest and during dipyridamole stress is significantly impaired in patients with hypertrophic cardiomyopathy [15], heart failure [21] and dilated cardiomyopathy [19].

Whether or not serum EPA levels correlated with the CFR of patients with known or suspected coronary artery disease (CAD) remains unclear. Therefore, the present study aimed to determine the relationship between serum EPA and CFR in patients with CAD using PC cine CMR. As the presence of significant coronary artery stenosis affects the CFR, we enrolled the CAD patients without $\geq 50 \%$ diameter stenosis on X-ray coronary angiography (CAG).

\section{Methods}

\section{Patients}

This study included 237 patients with known or suspected $\mathrm{CAD}$ who were assessed by X-ray coronary angiogram and cardiovascular magnetic resonance (CMR) including cine CMR, PC cine CMR, late gadolinium enhancement (LGE) CMR. Figure 1 illustrates flow chart of patient enrollment in this study. We excluded the patients with dilated cardiomyopathy $(n=10)$, severe valvular disease $(n=8)$, hypertrophic cardiomyopathy $(\mathrm{n}=7)$, sarcoidosis $(\mathrm{n}=3)$ and amyloidosis $(\mathrm{n}=1)$. We also excluded patients with history of coronary artery bypass graft surgery $(\mathrm{CABG})(\mathrm{n}=10)$ and patients who demonstrated significant coronary arterial stenoses on X-ray CAG $(\mathrm{n}=71)$. Finally, 127 patients (male, 116 (91\%); mean age, $72.2 \pm 7.4$ years) were enrolled in the present study. Table 1 summarizes the characteristics of the included patients. The medical histories of 42 (33\%), 64 (50\%) and $72(57 \%)$ of the patients included myocardial infarction, angina pectoris. We allocated the patients to groups with high $(\mathrm{n}=64 ; \mathrm{EPA} \geq 75.8 \mu \mathrm{g} / \mathrm{mL})$ and low $(\mathrm{n}=63$; EPA $<75.8 \mu \mathrm{g} / \mathrm{mL})$ median serum EPA. Other characteristics including coronary risk factors, cardiovascular history and medication did not significantly differ between the groups (Table 1). None of them were taking purified EPA. All patients provided written, informed consent to participate in this study, which was approved by the local institutional review board.

\section{CMR acquisition}

CMR was performed on a 1.5-T MR system equipped with 32 channel cardiac coils (Achieva, Philips Healthcare, Best, The Netherlands). All patients were assessed by cine CMR, PC cine CMR and LGE CMR. Imaging was performed after overnight fasting. Serum fatty acid measurement was performed on the same day as CMR.

\section{Cine CMR acquisition}

Vector-electrocardiographic (VCG) monitoring leads were positioned on supine patients and then imaging started. Scout images were acquired in three orthogonal planes for cardiac orientation. Vertical and horizontal long-axis cine CMR of the LV was acquired using a steady-state free precession (SSFP) sequence. The LV volume and mass were calculated from short-axis cine images of the LV acquired from the apex to the base (repetition time, $4.1 \mathrm{~ms}$; echo time, $1.7 \mathrm{~ms}$; flip angle, $55^{\circ}$; field of view, $350 \times 350 \mathrm{~mm}$; acquisition matrix, $128 \times 128$; slice thickness, $10 \mathrm{~mm}$; and number of phases per cardiac cycle, 20).

\section{Phase-contrast cine CMR acquisition}

Cine CMR in the axial plane was obtained through the atrioventricular groove to locate the coronary sinus (Figure 1). The imaging plane for blood flow measurement by PC cine CMR was positioned perpendicular to the coronary sinus $2 \mathrm{~cm}$ from the ostium of the coronary sinus. Phase-contrast cine CMR of the coronary sinus was acquired during suspended shallow breath-holding

237 patients known or suspected CAD who underwent X-ray CAG and CMR

Exclusion criteria

- Dilated cardiomyopathy $(n=10)$

- Severe valvular disease $(n=8)$

- Hypertrophic obstructive cardiomyopathy $(n=7)$

- Sardoidosis, amyloidosis $(n=4)$

- Post CABG ( $n=10)$

198 patients

Excluded $n=71$, significant coronary

arterial stenosis on $\mathrm{X}$-ray coronary

angiography

127 patients

Figure 1 Flow chart of enrollment of study population. CAD, coronary artery disease; CMR, cardiovascular magnetic resonance; CABG, coronary artery bypass graft surgery. 
Table 1 Patient characteristics

\begin{tabular}{|c|c|c|c|c|}
\hline & $\begin{array}{l}\text { All patients } \\
(n=127)\end{array}$ & $\begin{array}{l}\text { High EPA group } \\
\qquad(n=64)\end{array}$ & $\begin{array}{l}\text { Low EPA group } \\
\qquad(n=63)\end{array}$ & *P value \\
\hline Age & $71.6 \pm 7.8$ & $71.8 \pm 7.6$ & $70.1 \pm 9.1$ & 0.18 \\
\hline Male & $116(91 \%)$ & $60(94 \%)$ & $56(89 \%)$ & 0.12 \\
\hline Height, cm & $163.4 \pm 7.1$ & $162.9 \pm 7.7$ & $164.4 \pm 6.5$ & 0.29 \\
\hline Body weight, kg & $65.4 \pm 11.1$ & $65.9 \pm 11.7$ & $64.3 \pm 9.7$ & 0.51 \\
\hline Body mass index, $\mathrm{kg} / \mathrm{m}^{2}$ & $24.3 \pm 3.0$ & $24.6 \pm 3.2$ & $23.7 \pm 2.5$ & 0.69 \\
\hline Systolic BP, mmHg & $137.9 \pm 20.8$ & $135.7 \pm 19.2$ & $140.5 \pm 22.3$ & 0.24 \\
\hline Diastolic BP, mmHg & $70.7 \pm 12.6$ & $69.6 \pm 13.3$ & $71.8 \pm 11.9$ & 0.35 \\
\hline Heart rate, beats per minute & $67.9 \pm 12.4$ & $66.3 \pm 10.7$ & $69.6 \pm 13.8$ & 0.18 \\
\hline \multicolumn{5}{|l|}{ Cardiovascular history } \\
\hline Myocardial infarction & $42(33 \%)$ & $19(30 \%)$ & $23(37 \%)$ & 0.64 \\
\hline Angina pectoris & $64(50 \%)$ & $35(55 \%)$ & $29(46 \%)$ & 0.22 \\
\hline \multicolumn{5}{|l|}{ Coronary risk factors } \\
\hline Hypertension & $93(73 \%)$ & $47(73 \%)$ & $46(73 \%)$ & 0.88 \\
\hline Dyslipidemia & $92(72 \%)$ & $46(72 \%)$ & $46(73 \%)$ & 0.84 \\
\hline Diabetes Mellitus & $39(31 \%)$ & $20(31 \%)$ & $19(30 \%)$ & 0.89 \\
\hline Current smoking & $16(13 \%)$ & $9(14 \%)$ & $7(11 \%)$ & 0.48 \\
\hline Family history of CAD & $6(5 \%)$ & $3(5 \%)$ & $3(5 \%)$ & 0.92 \\
\hline Obesity & $41(32 \%)$ & $25(39 \%)$ & $16(25 \%)$ & 0.13 \\
\hline \multicolumn{5}{|l|}{ Blood test results } \\
\hline $\mathrm{EPA}, \mu \mathrm{g} / \mathrm{mL}$ & $85.3 \pm 53.9$ & $121.5 \pm 53.3$ & $48.4 \pm 16.9$ & $<0.001$ \\
\hline $\mathrm{DHA}, \mu \mathrm{g} / \mathrm{mL}$ & $139.1 \pm 54.4$ & $163.2 \pm 51.9$ & $109.0 \pm 35.2$ & $<0.001$ \\
\hline $\mathrm{AA}, \mu \mathrm{g} / \mathrm{mL}$ & $178.6 \pm 52.9$ & $171.0 \pm 47.3$ & $179.6 \pm 52.5$ & 0.34 \\
\hline Total cholesterol, mmol/L & $179.0 \pm 38.6$ & $183.1 \pm 36.7$ & $172.8 \pm 37.9$ & 0.12 \\
\hline LDL cholesterol, mmol/L & $101.9 \pm 26.7$ & $106.6 \pm 31.7$ & $100.2 \pm 27.5$ & 0.23 \\
\hline $\mathrm{HDL}$ cholesterol, mmol/L & $57.3 \pm 15.9$ & $66.1 \pm 64.0$ & $53.0 \pm 14.3$ & 0.12 \\
\hline Triglyceride, mmol/L & $179.0 \pm 38.6$ & $171.1 \pm 125.1$ & $157.6 \pm 105.6$ & 0.51 \\
\hline Hemoglobin, g/dL & $14.3 \pm 1.6$ & $14.3 \pm 1.4$ & $14.1 \pm 1.6$ & 0.58 \\
\hline $\mathrm{HbA} 1 \mathrm{c}, \%$ & $6.0 \pm 0.9$ & $6.0 \pm 1.0$ & $5.8 \pm 0.8$ & 0.21 \\
\hline $\mathrm{eGFR}, \mathrm{mL} / \mathrm{min} / 1.73 \mathrm{~m}^{2}$ & $65.7 \pm 14.0$ & $64.5 \pm 13.2$ & $65.6 \pm 13.6$ & 0.65 \\
\hline CRP, mg/dL & $0.30 \pm 1.0$ & $0.15 \pm 0.21$ & $0.42 \pm 1.37$ & 0.25 \\
\hline $\mathrm{BNP}, \mathrm{pg} / \mathrm{dl}$ & $53.3 \pm 65.0$ & $46.6 \pm 43.9$ & $45.9 \pm 63.2$ & 0.94 \\
\hline \multicolumn{5}{|l|}{ Medication use } \\
\hline Statin & $82(65 \%)$ & $41(64 \%)$ & $41(65 \%)$ & 0.86 \\
\hline Antiplatelet agent & $108(85 \%)$ & $52(81 \%)$ & $56(89 \%)$ & 0.91 \\
\hline Calcium channel blocker & 45 (35\%) & $22(34 \%)$ & $23(37 \%)$ & 0.99 \\
\hline$\beta$ blocker & $48(38 \%)$ & $21(33 \%)$ & $27(43 \%)$ & 0.26 \\
\hline ACE/ARB & $73(57 \%)$ & $33(52 \%)$ & $40(63 \%)$ & 0.17 \\
\hline
\end{tabular}

Values are presented as mean \pm standard deviation (SD).

* $P$ value represents significance of difference between low EPA group and high EPA group.

EPA, eicosapentaenoic acid; AA, arachidonic acid, DHA, docosahexaenoic acid; BP, blood pressure; CAD, coronary artery disease; LDL; low density lipoprotein; HDL, high density lipoprotein, eGFR, estimated glomerular filtration rate; CRP, C-reactive protein; BNP, brain natriuretic peptide; $A C E$, angiotensin converting enzyme inhibitor; ARB angiotensin receptor blocker. 
using a VCG triggered gradient echo sequence (repetition time, $7.3 \mathrm{~ms}$; echo time, $4.4 \mathrm{~ms}$; flip angle, $10^{\circ}$; field of view, $240 \times 194 \mathrm{~mm}$; acquisition matrix, $128 \times 128$; and number of phases per cardiac cycle, 20). Pharmacological stress was achieved by injecting ATP $\left(160 \mu \mathrm{g} \cdot \mathrm{kg}^{-1} \cdot \mathrm{min}^{-1}\right)$ into the left antecubital vein for $4 \mathrm{~min}$. PC cine CMR images of the coronary sinus were acquired during ATP stress and at rest. The duration between stress and resting image acquisition was at least $10 \mathrm{~min}$. All patients were asked to refrain from caffeinated beverages for at least 24 hours prior to CMR.

\section{Late gadolinium enhanced image acquisition}

After acquiring phase-contrast cine CMR, the patients were injected with $0.15 \mathrm{mmol} / \mathrm{kg}$ of gadopentetate dimeglumine (Magnevist, Bayer Healthcare, Leverkusen, Germany). Late gadolinium-enhanced CMR was obtained in the same planes as cine images 15 min later using an inversion recovery-prepared gradient-echo sequence. The imaging parameters for late gadolinium-enhanced imaging comprised: repetition time, $4.3 \mathrm{~ms}$; echo time, $1.3 \mathrm{~ms}$; flip angle, $15^{\circ}$; field of view, $380 \times 380 \mathrm{~mm}$; acquisition matrix, $256 \times 180$; and slice thickness, $10 \mathrm{~mm}$. The null point of the normal myocardium was determined using a LookLocker sequence.

\section{X-ray coronary angiography}

An observer who was blinded to the results of CMR interpreted the conventional X-ray CAG using quantitative software (QangioXA, Medis, Inc., Raleigh, North Carolina). Intracoronary administration of isosorbide dinitrate (2 to $3 \mathrm{mg}$ ) was performed in all patients before contrast injection. We performed quantitative coronary angiography (QCA) analysis for evaluating the degree of coronary arterial stenosis on X-ray CAG. In our trial, significant coronary arterial stenosis was defined as reduction in luminal diameter $\geq 50 \%$ by QCA analysis.

\section{Measurement of serum fatty acids}

Blood was sampled from all patients after an overnight fast for at least $12 \mathrm{~h}$. Serum levels of EPA, AA, and docosahexaenoic acid (DHA) were measured using a gas chromatograph (GC-2010; Shimadzu, Kyoto, Japan) and a capillary column (BPX90; Wako, Osaka, Japan) at a central laboratory (BML, Tokyo, Japan).

\section{Data analysis}

Two observers used a workstation (Extend MR WorkSpace, Philips Healthcare) to analyze the cine MR, PC cine $\mathrm{MR}$, and LGE images. All CMR images were rendered anonymous and reviewed in random order. To measure the LV cardiac mass, epi- and endocardial borders of the LV on the short axis cines were manually traced with exclusion of the papillary muscles at each anatomic level that encompassed the LV. The LV mass was calculated by the consensus of two observers as the sum of the myocardial volume areas multiplied by the specific gravity $(1.05 \mathrm{~g} / \mathrm{mL})$ of the myocardial tissue [22].

The contours of the coronary sinus were manually traced on each frame of all PC cine images to quantify blood flow in the coronary sinus and velocity in the adjacent tissue was measured for phase-offset correction. Blood flow in the coronary sinus was calculated by integrating the product of the cross-sectional area and the mean velocity in the coronary sinus and corrected using mean velocity in the adjacent tissue for all cardiac phases in the cardiac cycle. In line with other studies, we also corrected coronary sinus blood flow using rate pressure products (RPP) [16,19,23-26].

$$
\begin{aligned}
& \operatorname{RPP}(\mathrm{mmHg} \cdot \mathrm{bpm})= \text { Systolic blood pressure }(\mathrm{mmHg}) \\
& \times \text { Heart rate }(\text { beats } / \mathrm{min}) \\
& \text { Corrected CS flow }(\mathrm{mL} / \mathrm{min})=\mathrm{CS} \text { flow }(\mathrm{mL} / \mathrm{min})
\end{aligned}
$$$$
\text { /RPP }(\mathrm{mmHg} \cdot \mathrm{bpm}) \times 7500
$$

The $\triangle \mathrm{CS}$ flow and CFR were calculated as:

$$
\begin{array}{r}
\Delta \text { CS flow }(\mathrm{mL} / \mathrm{min})= \\
\text { Corrected CS flow during ATP } \\
\text { infusion }(\mathrm{mL} / \mathrm{min})-\text { Corrected } \\
\text { CS flow at rest }(\mathrm{mL} / \mathrm{min}) \\
\text { Coronary flow reserve }(\mathrm{CFR})= \\
\text { Corrected CS flow during } \\
\text { ATP infusion }(\mathrm{mL} / \mathrm{min}) \\
/ \text { Corrected CS flow at } \\
\text { rest }(\mathrm{mL} / \mathrm{min})
\end{array}
$$

The coronary vascular resistance (CVR) was calculated as follows.

$$
\begin{aligned}
& \operatorname{CVR}(\mathrm{mmHg} \cdot \mathrm{min} / \mathrm{mL})=\text { mean arterial pressure } \\
& \text { (MAP) }(\mathrm{mmHg}) / \text { corrected } \\
& \text { CS flow }(\mathrm{mL} / \mathrm{min}) \\
& \operatorname{MAP}(\mathrm{mmHg})=[\text { systolic BP }(\mathrm{mmHg})+2 \mathrm{x} \text { diastolic } \\
& \mathrm{BP}(\mathrm{mmHg})] / 3
\end{aligned}
$$

Late gadolinium-enhancement was visually assessed and interpreted by the consensus of two observers who were blinded to the patients' information. The mass with LGE was measured on short-axis slices using manual planimetry. The total mass of LGE was calculated by summing the late gadolinium-enhanced mass of all sections, and the ratio (\%) of LGE is expressed as:

$$
\begin{aligned}
\text { LGE }(\%)= & \text { Total LGE volume } / \text { Total LV wall volume } \\
& \times 100(\%)
\end{aligned}
$$

None of the patients had atypical enhancement defined as mid-myocardial or sub-epicardial enhancement with multifocal distribution. 


\section{Statistical analysis}

Data were statistically analyzed using SPSS software, version 17.0 (SPSS, Inc., Chicago, IL, USA). Continuous values are presented as means \pm standard deviation (SD). Skewed values are presented as medians with the interquartile range (IQR). Normality was determined by the Shapiro-Wilk test. Coronary flow reserve determined by CMR was compared between the high and low EPA groups. Differences between the groups were evaluated using an unpaired $t$-test for normally distributed variables, and the Mann-Whitney $U$ test for skewed variables. The relationship between serum fatty acids and CFR determined by CMR was determined using Pearson's correlation coefficient. Preserved CFR was defined as CFR $>2.5$ by PC cine CMR, which is the lower limit of normal flow reserve without obstructive CAD [27]. Independent predictors of impaired CFR were evaluated using univariate analysis and multivariate stepwise analyses. Inter- and intra-observer reproducibility was evaluated using the intraclass correlation coefficient (ICC). P values $<0.05$ were considered statistically significant. In addition, we allocated study population into 4 groups based on presence or absence of DM and serum EPA. We evaluated significance of difference between 4 groups by using one-way analysis of variance (ANOVA).

\section{Results}

\section{Cardiac CMR findings}

We acquired PC cine CMR from all patients in the resting state and during ATP stress (Figure 2). Figure 3 shows representative blood flow curves of the coronary sinus at rest and during infusion. Table 2 summarizes the CMR findings. Cine parameters such as LV end-diastolic volume index (LVEDVI), LV end-systolic volume index (LVESVI), LV ejection fraction (LVEF), LV mass index did not significantly differ between the low and high EPA groups. In addition, LGE parameters, such as presence of LGE and percentage of LGE (\%) also did not significantly differ between the groups.

\section{Comparison of coronary flow reserve in low and high EPA groups}

Table 3 compares coronary sinus blood flow at rest and during ATP infusion, and CFR in the low and high EPA/AA groups. The coronary sinus blood flow measurements were reproducible, with ICCs of 0.93 and 0.95 for inter- and intra-observer reproducibility, respectively. No significant difference between low and high EPA groups was found in HR, SBP, DBP and RPP at rest and during ATP infusion. SBP and DBP were significantly decreased by ATP infusion both in low and high EPA groups. Corrected coronary sinus blood flow was significantly augmented by ATP infusion in the low and high EPA groups (from 79.1 \pm 32.4 to $191.5 \pm 87.2 \mathrm{~mL} / \mathrm{min}, \mathrm{p}<0.001$ and from $82.3 \pm 35.0$ to $229.5 \pm 95.0 \mathrm{~mL} / \mathrm{min}, \mathrm{p}<0.001$, respectively), whereas $\triangle \mathrm{CS}$ flow was significantly lower in the group with low EPA $(112.4 \pm 73.7$ vs. $147.2 \pm 77.7 \mathrm{~mL} / \mathrm{min}, \mathrm{p}=0.036)$. Furthermore, CFR was significantly reduced in the low, compared with the high EPA group (2.54 \pm 1.00 vs. $2.91 \pm 0.98, p=0.038)$. We found significant difference in CVR during ATP infusion between high and low EPA groups $(0.53 \pm 0.25$ vs. $0.44 \pm 0.26, p=0.049)$. In addition, we divided study population into 4 groups based on presence or absence of DM and serum EPA (group 1, $\mathrm{n}=44$ : $\mathrm{DM}(-)$ \& EPA $\geq 75.8 \mu \mathrm{g} / \mathrm{mL}$, group 2, $\mathrm{n}=44: \mathrm{DM}(-)$ \& EPA $<75.8 \mu \mathrm{g} / \mathrm{mL}$, group $3, \mathrm{n}=20$ : $\mathrm{DM}(+)$ \& EPA $\geq 75.8 \mu \mathrm{g} / \mathrm{mL}$, group $4, \mathrm{n}=19$ : DM (+) \& EPA $<75.8 \mu \mathrm{g} / \mathrm{mL}$. CFR was highest in group 1 and lowest in group 4 (mean CFR: $3.04 \pm 1.00$ in group1, $2.69 \pm$ 1.11 in group $2,2.67 \pm 1.04$ in group $3,2.39 \pm 0.83$ ). However, difference between 4 group did not show significant difference ( $\mathrm{p}=0.16$ by one-way ANOVA).

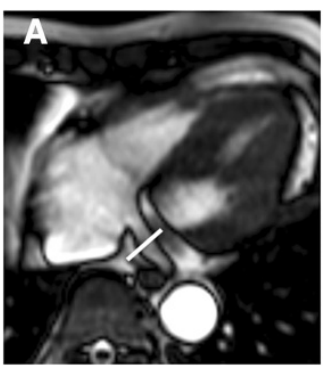

Cine MRI

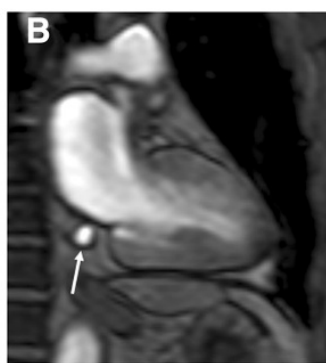

Magnitude image

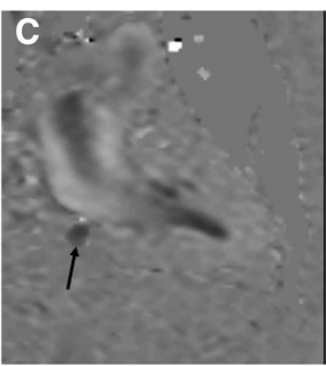

Phase contrast image

Phase contrast cine MRI

Figure 2 Phase-contrast cine cardiovascular magnetic resonance of the coronary sinus. (A) Axial image of the coronary sinus acquired by steady state free precession (white solid line). (B) Magnitude image of coronary sinus (white arrow). (C) Phase difference image of coronary sinus. Blood flow in the coronary sinus appears as a low signal intensity area in (C), (black arrow). CMR, cardiovascular magnetic resonance. 


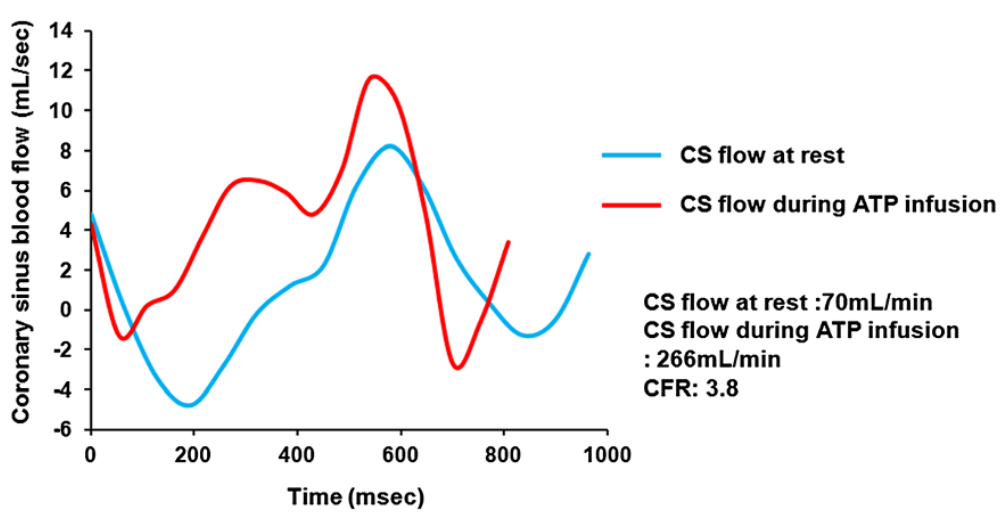

Figure 3 Curves of blood flow in coronary sinus. Blood flow was measured using phase-contrast cine CMR. Blue and red lines, blood flow at rest and during ATP infusion, respectively. Coronary sinus blood flow increased about 4-fold in this patient during ATP infusion (rest vs. ATP infusion: $=70$ vs. $266 \mathrm{~mL} / \mathrm{min}$; CFR = 3.8). ATP, adenosine triphosphate; CFR, coronary flow reserve; CS, coronary sinus.

Relationship of serum fatty acid and coronary flow reserve Figure 4 shows relationships between serum fatty acids (EPA, DHA, AA) and CFR evaluated by CMR. Serum levels of EPA and DHA correlated significantly and positively $(\mathrm{R}=0.35$ and $\mathrm{p}<0.001, \mathrm{R}=0.29$ and $\mathrm{p}=0.001$, and $\mathrm{R}=0.35$ and $\mathrm{p}<0.001$, respectively), whereas those of $\mathrm{AA}$ correlated significantly and negatively $(R=-0.18, \mathrm{p}=0.046)$ with CFR.

\section{Univariate and multivariate analyses of predictors of reduced CFR}

Table 4 shows the results of the univariate and multivariate analyses of predictors of CFR $>2.5$ determined by PC cine CMR. Univariate analysis selected history of diabetes mellitus, serum EPA, LVMI on cine CMR as significant predictors of CFR $>2.5$. Variables with $\mathrm{p}$ values $<0.05$ in the univariate analysis were included in the multiple stepwise regression models. Table 4 shows that the EPA was a significant determinant CFR $>2.5$ (odds ratio (OR), 1.01; 95\% confidence interval (CI), $1.00-1.02$; $\mathrm{p}=0.008$ ). Other independent variables comprised history of diabetes mellitus (OR, 0.39; 95\%CI, $0.17-0.89 ; \mathrm{p}=0.026$ ), LVMI (OR, 0.97; 95\%CI, $0.95-0.99 ; \mathrm{p}=0.024$ ).

\section{Discussion}

This is the first investigation of the relation between CFR evaluated as changes in coronary sinus flow induced by ATP infusion, and serum EPA. In patients without significant coronary arterial stenosis on X-ray coronary angiography, serum EPA and DHA levels significantly and positively correlated with CFR. Multivariate linear regression analysis showed that the EPA was an independent predictor of impairment of CFR evaluated by CMR. These results indicated that the serum EPA plays an important role in the regulation of CFR in patients with CAD.

Table 2 CMR findings

\begin{tabular}{|c|c|c|c|c|}
\hline & $\begin{array}{l}\text { All patients } \\
(n=127)\end{array}$ & $\begin{array}{l}\text { High EPA group } \\
\qquad(n=64)\end{array}$ & $\begin{array}{l}\text { Low EPA group } \\
\qquad(n=63)\end{array}$ & * P value \\
\hline \multicolumn{5}{|l|}{ Cine CMR } \\
\hline LVEDVI, $\mathrm{mL} / \mathrm{m}^{2}$ & $73.9 \pm 24.9$ & $71.7 \pm 21.8$ & $76.1 \pm 27.7$ & 0.33 \\
\hline LVESVI, mL/m² & $33.2 \pm 19.9$ & $31.0 \pm 19.1$ & $35.4 \pm 20.7$ & 0.21 \\
\hline LVSVI, mL/m² & $40.8 \pm 10.5$ & $40.8 \pm 8.6$ & $40.8 \pm 12.3$ & 0.98 \\
\hline LVEF, \% & $57.1 \pm 12.1$ & $58.9 \pm 11.7$ & $55.3 \pm 16.9$ & 0.094 \\
\hline LV mass index, $\mathrm{g} / \mathrm{m}^{2}$ & $56.3 \pm 16.9$ & $57.8 \pm 17.0$ & $54.8 \pm 16.9$ & 0.32 \\
\hline \multicolumn{5}{|c|}{ Late Gadolinium enhanced CMR } \\
\hline Presence of LGE & $66(52 \%)$ & $32(50 \%)$ & $34(54 \%)$ & 0.65 \\
\hline LGE (\%) & $10.6 \pm 14.2$ & $9.0 \pm 13.1$ & $12.2 \pm 15.2$ & 0.21 \\
\hline
\end{tabular}

Values are presented as mean \pm standard deviation (SD).

* $P$ value represents significance of difference between low EPA group and high EPA group.

EPA, eicosapentaenoic acid; CMR, cardiovascular magnetic resonance; LV, left ventricle; EDVI, end-diastolic volume index; ESVI, end-systolic volume index; SVI, stroke volume index; $E F$, ejection fraction; LGE, late gadolinium enhancement. 
Table 3 Coronary sinus blood flow at rest and during ATP infusion in groups with low and high EPA

\begin{tabular}{|c|c|c|c|c|}
\hline & All patients, $(\mathrm{N}=127)$ & High EPA group, $(n=64)$ & Low EPA group, $(n=63)$ & * P-value \\
\hline $\mathrm{HR}$ at rest, bpm & $68 \pm 12$ & $66 \pm 11$ & $70 \pm 14$ & 0.14 \\
\hline HR during ATP infusion, bpm & $71 \pm 12$ & $69 \pm 11$ & $73 \pm 12$ & 0.086 \\
\hline $\mathrm{SBP}$ at rest, $\mathrm{mmHg}$ & $138 \pm 21$ & $136 \pm 19$ & $140 \pm 22$ & 0.22 \\
\hline SBP during ATP infusion, $\mathrm{mmHg}$ & $117 \pm 20^{* *}$ & $117 \pm 19^{* *}$ & $117 \pm 22^{* *}$ & 1.00 \\
\hline $\mathrm{DBP}$ at rest, $\mathrm{mmHg}$ & $71 \pm 13$ & $69 \pm 13$ & $72 \pm 12$ & 0.33 \\
\hline DBP during ATP infusion, $\mathrm{mmHg}$ & $62 \pm 12^{* *}$ & $62 \pm 13^{* *}$ & $72 \pm 12^{* *}$ & 0.87 \\
\hline RPP at rest & $9432 \pm 2505$ & $9047 \pm 2206$ & $9825 \pm 2739$ & 0.11 \\
\hline RPP during ATP infusion & $8303 \pm 2105^{* *}$ & $8116 \pm 2111^{* *}$ & $8508 \pm 2100^{* *}$ & 0.34 \\
\hline cCS flow at rest $(\mathrm{mL} / \mathrm{min})$ & $77.4 \pm 32.7$ & $82.3 \pm 35.0$ & $79.1 \pm 32.4$ & 0.59 \\
\hline cCS flow during ATP infusion ( $\mathrm{mL} / \mathrm{min})$ & $203.3 \pm 89.6^{* *}$ & $229.5 \pm 95.0^{* *}$ & $191.5 \pm 87.2^{* *}$ & 0.021 \\
\hline$\Delta$ cCS flow $(\mathrm{mL} / \mathrm{min})$ & $125.9 \pm 75.8$ & $147.2 \pm 77.7$ & $112.4 \pm 73.7$ & 0.011 \\
\hline Coronary flow reserve & $2.75 \pm 1.03$ & $2.91 \pm 0.98$ & $2.54 \pm 1.00$ & 0.038 \\
\hline $\mathrm{CVR}$ at rest, $\mathrm{mmHg} \cdot \mathrm{min} / \mathrm{mL}$ & $1.37 \pm 0.54$ & $1.33 \pm 0.60$ & $1.41 \pm 0.47$ & 0.73 \\
\hline CVR during ATP infusion $\mathrm{mmHg} \cdot \mathrm{min} / \mathrm{mL}$ & $0.49 \pm 0.26^{* *}$ & $0.44 \pm 0.26^{* *}$ & $0.53 \pm 0.25^{* *}$ & 0.049 \\
\hline
\end{tabular}

Data are expressed as mean \pm SD. ${ }^{*}$ P-value represents significance of the difference between low EPA group and high EPA group. ${ }^{* *} \mathrm{P}<0.05$ vs. at rest. $\mathrm{HR}=$ heart rate, $\mathrm{ATP}=$ adenosine triphosphate. $\mathrm{SBP}=$ systolic blood pressure, $\mathrm{DBP}=$ diastolic blood pressure, RPP, rate pressure product; $\mathrm{ATP}$, adenosine triphosphate; EPA, eicosapentaenoic acid; $C S$, coronary sinus, CVR = coronary vascular resistance.

$\mathrm{cCS}$ flow $=$ corrected coronary sinus flow, $\triangle \mathrm{CCS}$ flow $=\mathrm{cCS}$ flow during ATP infusion $-\mathrm{cCS}$ flow at rest. Coronary flow reserve $=\mathrm{cCS}$ flow during ATP infusion $/ \mathrm{cCS}$ flow at rest $\times 100 . M A P=(S B P+D B P \times 2) / 3, C V R=M A P / c C S$ flow.

\section{The independent predictors and potential mechanism of impairment of CFR}

The results of multivariate regression analysis in this study revealed that presence of DM, LVMI and serum EPA were the independent predictors of preserved CFR $(\mathrm{CFR}>2.5)$. The CFR is an index of coronary microvascular function in patients without epicardial coronary arterial stenosis, therefore, we assessed the relationship between microvascular function and serum EPA concentration. It's well known that the women more frequently develop microvascular disease (MVD) in comparison to men, however, men and women who have coronary MVD often have conventional risk factors, such as hypertension and DM [28]. The presence of DM was an independent predictor of CFR in this study, and the result is in line with previous study.
However, presence of hypertension and presence of dyslipidemia were not independent predictors of CFR. The main reason is that the ACEI/ARB treatment for hypertension or statin treatment for dyslipidemia was already started. These drugs potentially have benefirial effects to MVD [28]. The serum EPA might play an important role for the regulation of CFR, and EPA drug treatment potentially improve the microvascular function in patients with MVD.

LV hypertrophy is another important mechanism of CFR impairment. presence of significant coronary arterial stenosis that results in dilatation already at rest. The patients with LV hypertrophy have a higher level of autoregulated blood flow at rest to meet the demand of an increased myocardial mass [29]. Therefore, CFR is reduced because resting flow is already increased closer to the
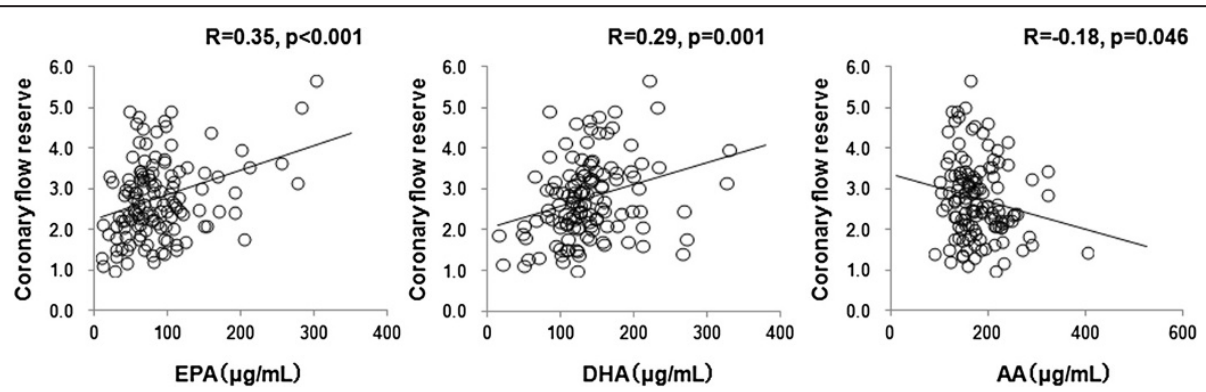

Figure 4 Relationship between serum fatty acid and coronary flow reserve evaluated by phase-contrast cine cardiovascular magnetic resonance. Serum levels of EPA and DHA significantly and positively correlate with CFR $(R=0.35, p<0.001 ; R=0.29, p=0.001)$. Serum $A A$ levels significantly and negatively correlate with CFR determined by PC cine CMR $(R=-0.18, p=0.046)$. CFR coronary flow reserve; DHA, docosahexaenoic acid; EPA, eicosapentaenoic acid. 
Table 4 Predictors of coronary flow reserve $>2.5$ determined by univariate and multivariate analyses

\begin{tabular}{|c|c|c|c|c|c|c|}
\hline & \multicolumn{3}{|c|}{ Univariate analysis } & \multicolumn{3}{|c|}{ Multivariate analysis } \\
\hline & Odds ratio & $95 \% \mathrm{Cl}$ & $P$ value & Odds ratio & $95 \% \mathrm{Cl}$ & $P$ value \\
\hline \multicolumn{7}{|l|}{ Patients' characteristics } \\
\hline Age, per year & 1.01 & $0.97-1.05$ & 0.65 & Not selected & & \\
\hline Male, men & 1.58 & $0.43-5.68$ & 0.49 & Not selected & & \\
\hline Systolic blood pressure, per mmHg & 0.99 & $0.97-1.01$ & 0.99 & Not selected & & \\
\hline Diastolic blood pressure, per $\mathrm{mmHg}$ & 0.98 & $0.95-1.02$ & 0.31 & Not selected & & \\
\hline Heart rate. Per bpm & 0.98 & $0.95-1.01$ & 0.19 & Not selected & & \\
\hline History of myocardial infarction, yes & 1.27 & $0.57-2.86$ & 0.55 & Not selected & & \\
\hline History of angina pectoris, yes & 1.56 & $0.71-3.56$ & 0.26 & Not selected & & \\
\hline \multicolumn{7}{|l|}{ Coronary risk factors } \\
\hline Hypertension, yes & 0.69 & $0.26-0.69$ & 0.45 & Not selected & & \\
\hline Dyslipidemia, yes & 0.67 & $0.25-1.77$ & 0.42 & Not selected & & \\
\hline Diabetes Mellitus, yes & 0.35 & $0.16-0.77$ & 0.009 & 0.39 & $0.17-0.89$ & 0.026 \\
\hline Current smoking, yes & 1.49 & $0.50-4.45$ & 0.48 & Not selected & & \\
\hline Family history of CAD, yes & 1.21 & $0.23-6.40$ & 0.82 & Not selected & & \\
\hline Obesity, BMI $\geq 25 \mathrm{~kg} / \mathrm{m}^{2}$, yes & 1.41 & $0.66-3.00$ & 0.37 & Not selected & & \\
\hline \multicolumn{7}{|l|}{ Blood testing } \\
\hline EPA, per $\mu \mathrm{g} / \mathrm{mL}$ & 1.01 & $1.00-1.02$ & 0.017 & 1.01 & $1.00-1.02$ & 0.008 \\
\hline Total cholesterol, per mmol/L & 1.00 & $0.99-1.01$ & 0.62 & Not selected & & \\
\hline LDL cholesterol, per mmol/L & 1.00 & $0.99-1.01$ & 0.93 & Not selected & & \\
\hline HDL cholesterol, per mmol/L & 1.02 & $0.99-1.04$ & 0.21 & Not selected & & \\
\hline Triglyceride, per mmol/L & 1.00 & $0.99-1.00$ & 0.97 & Not selected & & \\
\hline HbA1c, per\% & 0.92 & $0.63-1.34$ & 0.68 & Not selected & & \\
\hline eGFR, per $\mathrm{mL} / \mathrm{min} / 1.73 \mathrm{~m}^{2}$ & 0.99 & $0.97-1.03$ & 0.94 & Not selected & & \\
\hline CRP, per mg/dL & 0.57 & $0.18-1.80$ & 0.34 & Not selected & & \\
\hline BNP, per pg/dl & 1.00 & $0.99-1.01$ & 0.66 & Not selected & & \\
\hline \multicolumn{7}{|l|}{ Cine CMR } \\
\hline LVEDVI, per $\mathrm{mL} / \mathrm{m}^{2}$ & 1.00 & $0.99-1.01$ & 0.75 & Not selected & & \\
\hline LVESVI, per mL/m² & 0.99 & $0.98-1.01$ & 0.32 & Not selected & & \\
\hline LVEF, per\% & 1.03 & $1.00-1.06$ & 0.066 & Not selected & & \\
\hline LVMI, per $\mathrm{g} / \mathrm{m}^{2}$ & 0.97 & $0.95-1.00$ & 0.019 & 0.97 & $0.95-0.99$ & 0.024 \\
\hline \multicolumn{7}{|l|}{ Late Gadolinium enhanced CMR } \\
\hline Presence of $L G E$, yes & 0.65 & $0.32-1.32$ & 0.24 & Not selected & & \\
\hline LGE (\%), per\% & 0.97 & $0.95-1.02$ & 0.35 & Not selected & & \\
\hline
\end{tabular}

The variables with $p$ value $<0.05$ in the univariate analysis were included in the multivariate analysis.

$\mathrm{Cl}$, confidence interval; CFR, coronary flow reserve; LGE, late gadolinium enhancement; BMI, body mass index; EPA, eicosapentaenoic acid; LDL; low density lipoprotein; $\mathrm{HDL}$, high density lipoprotein, eGFR, estimated glomerular filtration rate; CRP, C-reactive protein; BNP, brain natriuretic peptide; CMR, cardiovascular magnetic resonance; LV, left ventricle; EDVI, end-diastolic volume index; ESVI, end-systolic volume index; SVI, stroke volume index; EF, ejection fraction; LVMI, left ventricular mass index.

maximal level. The LVMI was an independent predictor of CFR in the current study, which is in line with previous study.

\section{Clinical significance of serum fatty acid on CAD prevention} Epidemiological studies have shown that the incidence of acute myocardial infarction is substantially lower among non-emigrated inhabitants of Greenland (5.3\%), than in individuals from the United States (40.4\%) and Denmark (34.7\%), with age- and sex adjusted death rates for ischemic heart disease [30]. This is mainly due to the composition of Eskimo food in north western Greenland, which is dominated by n-3 PUFA, such as EPA and DHA (ratios of n-3 PUFA in total Eskimo and Danish dietary 
intake of fatty acids: $13.7 \%$ and $2.8 \%$, respectively) [30]. The randomized controlled GISSI-Prevenzione trial showed that dietary n-3 PUFA intake can reduce the rate of cardiovascular death among patients after a recent ( $\leq 3$ months) myocardial infarction [13]. The large, randomized JELIS control trial found that that administering pure EPA together with statin therapy reduced the risk of developing coronary events to $19 \%$ [14]. However, precisely how n-3 PUFA prevents CAD remains unknown. Some reports have shown favorable effects of n-3 PUFA on the cardiovascular system. Leaf et al. showed that n-3 PUFA helped to prevent arrhythmic deaths, including sudden cardiac death [31]. Ghio et al. showed that n-3 PUFA significantly increases LV systolic function in patients with symptomatic heart failure of any etiology [31]. In addition, Moertl et al. found that n-3 PUFA administered for 3 months exerts a dose-dependent increase in the LVEF of patients with chronic heart failure. Moreover, high-dose n-3 PUFA intervention significantly improves endothelial function and decreases interleukin 6 levels [32]. We found that the serum EPA is an independent predictor of impaired CFR evaluated by PC cine CMR. This finding indicates that improved CFR might be an important mechanism through which n-3 PUFA prevents cardiovascular events.

\section{Clinical implications}

In this study, we enrolled the CAD patients without $\geq 50 \%$ diameter stenosis on X-ray CAG, and assessed the microvascular function by calculating the CFR by PC-cine CMR. It is important to evaluate the disease severity in patients with non-obstructive $\mathrm{CAD}$, because the prognosis of MVD is not as benign as commonly thought [28]. Randomized placebo-controlled studies have demonstrated that angiotensin-converting enzyme inhibitors, statins etc. relieve symptoms, vascular dysfunction, or both [28]. In the current study, most of our patients were administered with aspirin and statins, and had relatively low LDL cholesterol levels. Regardless, the EPA significantly correlated with CFR, and it was an independent predictor of reduced CFR. These results suggest that EPA therapy will help to improve CFR and prevent cardiovascular events through different mechanisms from statins or antiplatelet drugs. A further prospective interventional study is required to clarify this point.

\section{Study limitation}

First, this was a single center study with a relatively small patient cohort. Although CFR assessment by PC cine CMR is non-invasive and useful, CMR is contraindicated for patients implanted with pacemakers or cardiovascular defibrillators and those with claustrophobia. Such patients were excluded from the present study. Second, we excluded the CAD patients with significant coronary arterial stenosis (diameter reduction $\geq 50 \%$ by QCA analysis). Therefore, the findings of this study can only be applied to the CAD patients without $\geq 50 \%$ diameter stenosis. The reason to exclude the patients with significant CAD was that the fall of blood pressure induced by ATP should have too much compromised coronary flow with the risk to produce an unwanted myocardial ischemia. Third, CVR assessed in the current study mean the resistance throughout the entire cardiac cycle, included the systolic period during which a compressive resistance is added to the resistance due to the coronary vasomotor tone, usually indicated as coronary "vascular" resistance (auto-regulative resistance).

\section{Conclusion}

Serum EPA is significantly correlated with CFR in CAD patients without significant coronary artery stenosis.

\section{Competing interests}

The authors declare that they have no competing interests.

\section{Authors' contributions}

SK designed the study protocol, carried out the MR studies, analyzed the data and drafted the manuscript. KF and JK analyzed the data and drafted the manuscript. NI, MK, YK, IK, TN, TN, YT, KU, KK and SU helped to draft the manuscript. All authors read and approved the final manuscript.

\section{Acknowledgements}

We are grateful to Hiroshi Hayashi, RT and Yukihiro Ishii, RT (Department of Radiology, Kanagawa Cardiovascular and Respiratory Center) for technical assistance.

\section{Author details}

'Department of Cardiology, Kanagawa Cardiovascular and Respiratory Center, 6-16-1 Tomiokahigashi, Kanazawa-ku, Yokohama, Kanagawa 236-0051, Japan. 2Department of Endocrinology and Metabolism, Yokohama City University Hospital, Yokohama, Japan. ${ }^{3}$ Department of Cardiology, Yokohama City University Hospital, Yokohama, Japan. ${ }^{4}$ Division of Cardiology, Yokohama City University Medical Center, Yokohama, Japan.

Received: 17 July 2013 Accepted: 11 December 2013 Published: 20 December 2013

\section{References}

1. Kromhout D, Bosschieter EB, de Lezenne CC. The inverse relation between fish consumption and 20-year mortality from coronary heart disease. N Engl J Med. 1985; 312:1205-9.

2. Siscovick DS, Raghunathan TE, King I, Weinmann S, Wicklund KG, Albright J, Bovbjerg V, Arbogast P, Smith H, Kushi LH, et al. Dietary intake and cell membrane levels of long-chain $n-3$ polyunsaturated fatty acids and the risk of primary cardiac arrest. JAMA. 1995; 274:1363-7.

3. Daviglus ML, Stamler J, Orencia AJ, Dyer AR, Liu K, Greenland P, Walsh MK, Morris D, Shekelle RB. Fish consumption and the 30-year risk of fatal myocardial infarction. N Engl J Med. 1997; 336:1046-53.

4. Albert CM, Hennekens CH, O'Donnell CJ, Ajani UA, Carey VJ, Willett WC, Ruskin JN, Manson JE. Fish consumption and risk of sudden cardiac death. JAMA. 1998; 279:23-8.

5. Albert CM, Campos H, Stampfer MJ, Ridker PM, Manson JE, Willett WC, Ma J. Blood levels of long-chain n-3 fatty acids and the risk of sudden death. N Engl J Med. 2002; 346:1113-8.

6. Hu FB, Bronner L, Willett WC, Stampfer MJ, Rexrode KM, Albert CM, Hunter D, Manson JE. Fish and omega-3 fatty acid intake and risk of coronary heart disease in women. JAMA. 2002; 287:1815-21.

7. Lemaitre RN, King IB, Mozaffarian D, Kuller LH, Tracy RP, Siscovick DS. N-3 polyunsaturated fatty acids, fatal ischemic heart disease, and nonfatal 
myocardial infarction in older adults: the cardiovascular health study. Am J Clin Nutr. 2003; 77:319-25.

8. Harris WS, Park Y, Isley WL. Cardiovascular disease and long-chain omega-3 fatty acids. Curr Opin Lipidol. 2003; 14:9-14.

9. Din JN, Newby DE, Flapan AD. Omega 3 fatty acids and cardiovascular disease-fishing for a natural treatment. BMJ. 2004; 328:30-5.

10. Hirai A, Terano T, Hamazaki T, Sajiki J, Kondo S, Ozawa A, Fujita T, Miyamoto T, Tamura Y, Kumagai A. The effects of the oral administration of fish oil concentrate on the release and the metabolism of [14c]arachidonic acid and [14c]eicosapentaenoic acid by human platelets. Thromb Res. 1982; 28:285-98.

11. Tamura Y, Hirai A, Terano T, Takenaga M, Saitoh H, Tahara K, Yoshida S. Clinical and epidemiological studies of eicosapentaenoic acid (epa) in japan. Prog Lipid Res. 1986; 25:461-6.

12. Kawano H, Yano T, Mizuguchi K, Mochizuki H, Saito Y. Changes in aspects such as the collagenous fiber density and foam cell size of atherosclerotic lesions composed of foam cells, smooth muscle cells and fibrous components in rabbits caused by all-cis-5, 8, 11, 14, 17-icosapentaenoic acid. J Atheroscler Thromb. 2002; 9:170-7.

13. GISSI-Prevenzione Investigators (Gruppo Italiano per lo Studio della Sopravvivenza nell'Infarto miocardico). Dietary supplementation with $n-3$ polyunsaturated fatty acids and vitamin e after myocardial infarction: results of the gissi-prevenzione trial. Gruppo italiano per lo studio della sopravvivenza nell'infarto miocardico. Lancet. 1999; 354:447-55.

14. Yokoyama M, Origasa H, Matsuzaki M, Matsuzawa Y, Saito Y, Ishikawa Y, Oikawa S, Sasaki J, Hishida H, Itakura H, Kita T, Kitabatake A, Nakaya N, Sakata T, Shimada K, Shirato K. Effects of eicosapentaenoic acid on major coronary events in hypercholesterolaemic patients (jelis): a randomised open-label, blinded endpoint analysis. Lancet. 2007; 369:1090-8.

15. Kawada N, Sakuma H, Yamakado T, Takeda K, Isaka N, Nakano T, Higgins CB. Hypertrophic cardiomyopathy: Mr measurement of coronary blood flow and vasodilator flow reserve in patients and healthy subjects. Radiology. 1999; 211:129-35.

16. Schwitter J, DeMarco T, Kneifel S, von Schulthess GK, Jorg MC, Arheden H, Ruhm S, Stumpe K, Buck A, Parmley WW, Luscher TF, Higgins CB. Magnetic resonance-based assessment of global coronary flow and flow reserve and its relation to left ventricular functional parameters: a comparison with positron emission tomography. Circulation. 2000; 101:2696-702.

17. van Rossum AC, Visser FC, Hofman MB, Galjee MA, Westerhof N, Valk J. Global left ventricular perfusion: noninvasive measurement with cine $\mathrm{mr}$ imaging and phase velocity mapping of coronary venous outflow. Radiology. 1992; 182:685-91.

18. Lund GK, Wendland MF, Shimakawa A, Arheden H, Stahlberg F, Higgins CB, Saeed M. Coronary sinus flow measurement by means of velocity-encoded cine $\mathrm{mr}$ imaging: Validation by using flow probes in dogs. Radiology. 2000; 217:487-93.

19. Watzinger N, Lund GK, Saeed M, Reddy GP, Araoz PA, Yang M, Schwartz AB, Bedigian M, Higgins CB. Myocardial blood flow in patients with dilated cardiomyopathy: quantitative assessment with velocity-encoded cine magnetic resonance imaging of the coronary sinus. J Magn Reson Imaging. 2005; 21:347-53.

20. Arheden $H$, Saeed M, Tornqvist E, Lund G, Wendland MF, Higgins CB, Stahlberg F. Accuracy of segmented mr velocity mapping to measure small vessel pulsatile flow in a phantom simulating cardiac motion. J Magn Reson Imaging. 2001; 13:722-8.

21. Lund GK, Watzinger N, Saeed M, Reddy GP, Yang M, Araoz PA, Curatola D, Bedigian M, Higgins CB. Chronic heart failure: global left ventricular perfusion and coronary flow reserve with velocity-encoded cine $\mathrm{mr}$ imaging: initial results. Radiology. 2003; 227:209-15.

22. Semelka RC, Tomei E, Wagner S, Mayo J, Kondo C, Suzuki J, Caputo GR, Higgins CB. Normal left ventricular dimensions and function: interstudy reproducibility of measurements with cine mr imaging. Radiology. 1990; 174:763-8.

23. Siegrist PT, Gaemperli O, Koepfli P, Schepis T, Namdar M, Valenta I, Aiello F, Fleischmann S, Alkadhi H, Kaufmann PA. Repeatability of cold pressor test-induced flow increase assessed with $\mathrm{h}(2)(15) \mathrm{o}$ and pet. $J \mathrm{NuCl}$ Med. 2006; 47:1420-6.

24. Naya M, Tsukamoto T, Morita K, Katoh C, Furumoto T, Fujii S, Tamaki N, Tsutsui H. Olmesartan, but not amlodipine, improves endothelium-dependent coronary dilation in hypertensive patients. J Am Coll Cardiol. 2007; 50:1144-9.

25. Furuyama H, Odagawa $Y$, Katoh C, I wado $Y$, Yoshinaga $K$, Ito $Y$, Noriyasu $K$, Mabuchi M, Kuge Y, Kobayashi K, Tamaki N. Assessment of coronary function in children with a history of kawasaki disease using (15)o-water positron emission tomography. Circulation. 2002; 105:2878-84.

26. Alexanderson E, Rodriguez-Valero M, Martinez A, Calleja R, Lamothe PA, Sierra C, Garcia-Rojas L, Talayero JA, Cruz P, Meave A, Alexanderson G. Endothelial dysfunction in recently diagnosed type 2 diabetic patients evaluated by pet. Mol Imaging Biol. 2009; 11:1-5.

27. Vaccarino V, Khan D, Votaw J, Faber T, Veledar E, Jones DP, Goldberg J, Raggi P, Quyyumi AA, Bremner JD. Inflammation is related to coronary flow reserve detected by positron emission tomography in asymptomatic male twins. J Am Coll Cardiol. 2011; 57:1271-9.

28. Bugiardini R, Bairey Merz CN. Angina with "Normal" coronary arteries: a changing philosophy. JAMA. 2005; 293:477-84.

29. Hoffman Jl. A critical view of coronary reserve. Circulation. 1987; 75:16-11.

30. Dyerberg J, Bang HO. A hypothesis on the development of acute myocardial infarction in greenlanders. Scand J Clin Lab Invest Suppl. 1982; 161:7-13.

31. Leaf A, Kang JX, Xiao YF, Billman GE. Clinical prevention of sudden cardiac death by $n-3$ polyunsaturated fatty acids and mechanism of prevention of arrhythmias by $\mathrm{n}-3$ fish oils. Circulation. 2003; 107:2646-52.

32. Moertl D, Hammer A, Steiner S, Hutuleac R, Vonbank K, Berger R. Dose-dependent effects of omega-3-polyunsaturated fatty acids on systolic left ventricular function, endothelial function, and markers of inflammation in chronic heart failure of nonischemic origin: a double-blind, placebo-controlled, 3-arm study. Am Heart J. 2011; 161:915. e911-919.

doi:10.1186/1532-429X-15-106

Cite this article as: Kato et al:: Relationship between coronary flow reserve evaluated by phase-contrast cine cardiovascular magnetic resonance and serum eicosapentaenoic acid. Journal of Cardiovascular Magnetic Resonance 2013 15:106.

\section{Submit your next manuscript to BioMed Central and take full advantage of:}

- Convenient online submission

- Thorough peer review

- No space constraints or color figure charges

- Immediate publication on acceptance

- Inclusion in PubMed, CAS, Scopus and Google Scholar

- Research which is freely available for redistribution
C Biomed Central 